\title{
Conjuntivitis recurrente unilateral: Reporte de caso
}

\author{
*Jenny Kam Lin Ku Lozano ${ }^{1,3}$, Margarita Samudio Acevedo², Eduardo Maron Ku Lu,3 \\ ${ }^{1}$ Instituto Peruano de Oftalmología, Piura, Perú \\ ${ }^{2}$ Universidad Nacional de Asunción, Instituto de Investigaciones en Ciencias de la Salud. Paraguay \\ ${ }^{3}$ Centro Visión, Piura, Perú
}

Cómo referenciar este artículo/ How to reference this article:
Ku Lozano JK, Samudio Acevedo M, Ku Lu E. Conjuntivitis recurrente unilateral: Reporte de caso. Mem. Inst. Investig. Cienc. Salud. 2019; 17(3): 79-81

\section{RE S U M E N}

Paciente de 35 años, de sexo femenino, acudió a la clínica oftalmológica por conjuntivitis recurrente unilateral de aproximadamente dos meses de evolución, previamente tratada por dos oftalmólogos con antibióticos tópicos y lubricantes oculares, con alivio transitorio sintomático. Al realizar el examen clínico-oftalmológico se encontró un cuerpo extraño biológico. Se decidió extraerlo e iniciar tratamiento antibiótico, tras lo cual la paciente evolucionó favorablemente y se resolvió el cuadro.

Palabras clave: conjuntivitis unilateral recurrente, cuerpo extraño biológico.

\section{Recurrent unilateral conjunctivitis: case report}

\section{A B S T R A C T}

A 35-year-old woman was referred to the eye clinic with a 2-month history of recurrent unilateral conjunctivitis, managed previously by two ophthalmologists with topical antibiotics and ocular lubricants that provided symptomatic transient relief. Clinicalophthalmological examination revealed a biological foreign body which was removed and antibiotic treatment started with which the patient evolved favorably and the symptoms resolved.

Keywords: Recurrent unilateral conjunctivitis, biological foreign body.

\section{INTRODUCCIÓN}

La conjuntivitis recurrente unilateral es una patología poco frecuente y hay escasos reportes al respecto. Su etiología es variable, pero se ha descrito que una de las causas más frecuentes es la presencia de un cuerpo extraño, por lo que debe ser la principal sospecha en estos $\operatorname{casos}^{(1,2)}$. Asimismo, en Perú no se han reportado casos similares por lo que se realiza el presente reporte.

\section{Presentación del caso}

Paciente de 35 años de edad, sexo femenino, procedente de La Arena (Piura, Perú), que acude a consulta por presentar ojo rojo derecho asociado a sensación de cuerpo extraño y secreción purulenta de aproximadamente dos meses de evolución. Añade que fue vista previamente por dos oftalmólogos, quienes le indican tratamiento médico tópico (antibióticos y lubricantes), con lo que remitían parcialmente y de manera intermitente las molestias. Al momento de la consulta no se encontraba recibiendo tratamiento alguno. Refiere antecedente de leucoma de etiología desconocida (no recuerda hechos) desde la niñez en dicho ojo (OD). Niega cirugías, alergias, traumatismos. 


\section{Examen Oftalmológico}

Se observa exotropia 15 Dp en Ojo derecho (OD), con agudeza visual (sin corrección) en el OD: movimiento de manos, OS: 20/200. La presión intraocular en el OD de $15 \mathrm{mmHg}$ y en el OS: $12 \mathrm{mmHg}$.

En la biomicroscopía y fondo de ojo con lámpara de hendidura, en el OD se observa hiperemia conjuntival, secreción purulenta abundante y leucoma vascularizado que compromete el eje visual. No tiñe con Fluoresceína (ver Figura 1A). En la biomicroscopia y fondo de ojo con lámpara de hendidura del OI no se observan alteraciones.

Al examinar detalladamente las conjuntivas palpebrales y fondos de saco, se encontró un cuerpo extraño biológico en fondo de saco de párpado inferior (Figura 1B y 1C) decidiéndose extraer el mismo con un fórceps previa instilación de anestésico tópico (alcaine 1\%). Tras extracción se inició tratamiento antibiótico. Posteriormente, se envía cuerpo extraño biológico al laboratorio, determinándose que se trataba de una mosca doméstica (Fannia canicularis). La paciente evoluciona favorablemente con resolución definitiva del cuadro.

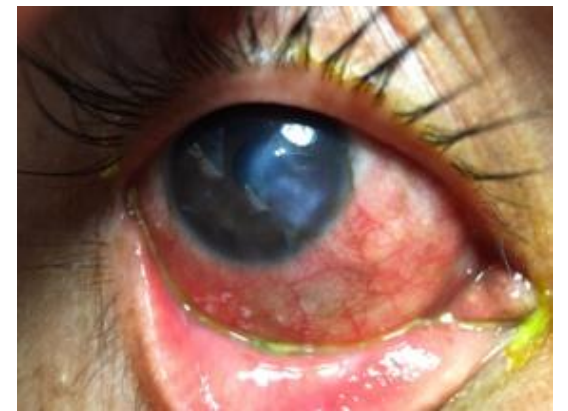

A

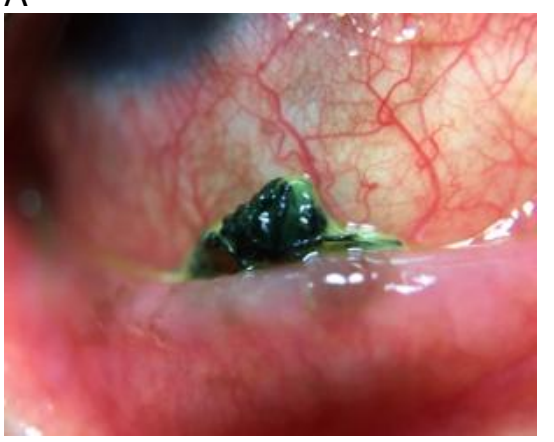

$\mathrm{C}$

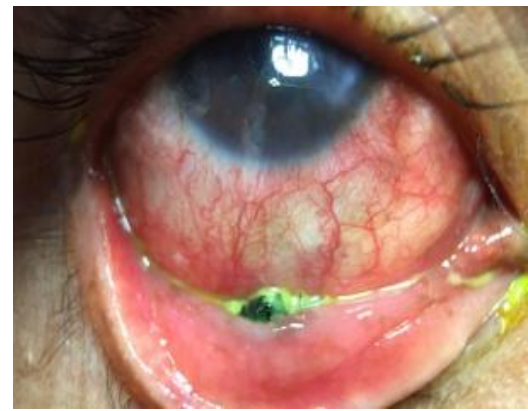

B

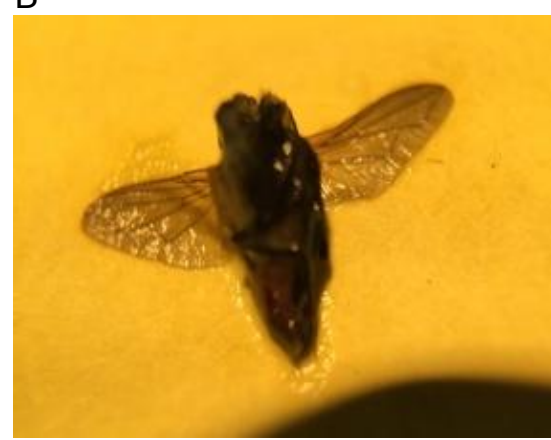

D

Figura 1: A. Ojo derecho sin tinción con fluoresceína. B: Cuerpo extraño biológico en fondo de saco OD. C. Cuerpo extraño biológico en fondo de saco OD (con aumento). D. Mosca doméstica (Fannia canicularis)

\section{DISCUSIÓN}

Se han descrito casos de retención de cuerpos extraños animados e inanimados que pueden producir o no daño corneal, conjuntivitis o dermatitis periorbitaria ${ }^{(1,2)}$ dependiendo de si éstos son tóxicos (objetos inanimados) o liberan alguna sustancia tóxica (insectos) ${ }^{(1-4)}$.

El tamaño es independiente de la sintomatología, ya que se ha descrito estudios donde el cuerpo extraño llegaba hasta los $8 \mathrm{~mm}^{(5,7)}$. De igual manera ocurre con el tiempo, ya que incluso se ha descrito un caso de retención por 28 años (vidrio) ${ }^{(4,5)}$.

El sitio de retención más frecuente es el fórnix conjuntival superior ya que el músculo orbicular puede interactuar con el tarso superior para atrapar cuerpos extraños y al mantener esta ubicación, se evita la irritación corneal ${ }^{(5-8)}$ aunque también se han descrito en la placa tarsal ${ }^{(9,10)}$.

En nuestro caso, la paciente presentaba un leucoma en ojo derecho, cuyo antecedente pudo haber producido una disminución de la sensibilidad corneal, favoreciendo la persistencia del cuerpo extraño por más de un mes en el fondo de saco inferior de dicho ojo. 
Siendo la conjuntivitis unilateral recurrente una patología poco frecuente y con sintomatología inespecífica es crucial una detallada historia clínica y un óptimo examen clínico-oftalmológico para poder determinar la causa ${ }^{(11-13)}$.

En conclusión se debe sospechar la presencia de cuerpo extraño ante un caso de conjuntivitis recurrente unilateral refractaria a tratamiento especializado y realizar una detallada historia clínica y un examen clínico-oftalmológico exhaustivo y minucioso a cada paciente, sobre todo a los casos recurrentes para lograr un diagnóstico oportuno y manejo apropiado inmediato.

Conflictos de intereses: los autores declaran no tener conflictos de interés.

Aspectos éticos: Se obtuvo consentimiento informado de la paciente para la presentación de la información contenida en el artículo.

\section{REFERENCIAS BIBLIOGRÁFICAS}

1. Monestam E, Bjornstig U. Eye injuries in northern Sweden. Acta Ophthalmol 1991;69: 1-5.

2. Chung R, Chew R, Au-Eong K. Coleoptera cincindelidae beetle in the eye. Singapore Med J 2005; 46(10):564-5.

3. Taneri S, Wagner E, Busse H. Lidschwel lung und Somnolenz nach Heugabelstoß ins Auge. Ophthalmologe 2001; 98: 492-3.

4. Meyer D, Tangas C. Recurrent unilateral conjunctivitis: a 24 year old occult foreign body is recovered. Clin Exp Ophthalmol 2003;31:455-6.

5. Carroll M. Retained glass foreign body in the eyelid. Am J Ophthalmol 1989; 107:555-6.

6. Gerding $\mathrm{H}$. Unusually Long Foreign. Klin Monatsbl Augenheilkd 2013;230:390-1.

7. Bhatt $P$, Lam F, Roberts F. Peripheral ulcerative keratitis due to a 'long lost' hard contact lens. Clin Experiment Ophthalmol 2007;35:550-2.

8. Sakata C, Hiraoka T, Oshika T. Unusually large plastic toy as a persisting conjunctival foreign body. Jpn J Ophthalmol 2007;51:231-3.
9. Wolter J, Peterson N. A wild rice seed under the upper eyelid: found after 14 months by eversion. Mich Med 1973; 72: 835-6.

10. Kalavathy C, Parmar P, Kaliamurthy J, Jesudasan C. Keratoconjunctivitis caused by an unusual retained conjunctival foreign body: A frequently unrecognized entity. Indian J Ophthalmol 2014;62:633-5.

11. Kanski JJ, Bowling B, eds. Clinical ophthalmology, a systematic approach. 7th Ed. London: Elsevier, 2011: 131-58.

12. Sen E, Elgin U, Koc F, Öztürk F. A $1.5 \mathrm{~cm}$ long unknown subconjunctival grass inflorescence misdiagnosed as relapsing conjunctivitis for one year. Turk J Pediatr 2011; 53:699-701.

13. Rostron E, Abdelaziz L, Barbara R, Metcalfe T. Missed sclera buckle as a cause of recurrent conjunctivitis. BMJ Case Reports 2012; 2012. pii: bcr2012006931. 